\title{
Canasta básica alimentaria e índice de precios en Santander, Colombia, 1999-2000
}

\author{
O scar Fernando Herrán-Falla, M en C, ${ }^{(1)}$ Gloria Esperanza Prada-Gómez, N utr, ${ }^{(1)}$ \\ Gonzalo Alberto Patiño-Benavides, PHD, Ec.(2)
}

Herrán-Falla OF, Prada-Gómez GE, Patiño-Benavides GA. Canasta básica alimentaria e índice de precios en Santander, Colombia, 1999-2000. Salud Publica Mex 2003;45:35-42. El texto completo en inglés de este artículo está disponible en: http://www.insp.mx/salud/index.html

\section{Resumen}

Objetivo. Establecer indicadores de seguridad alimentaria (SA) en dos municipios colombianos. Material y métodos. En el periodo 1999-2000 se realizó un estudio descriptivo en dos municipios del departamento de Santander, Colombia, que determinó el costo de canastas básicas alimentarias (C BA), para estimar índices de precios (IPC), su variación, e indicadores de SA relacionados con el salario mínimo legal vigente (SMLV). Se calcularon estadísticos de tendencia central y dispersión según el tipo de variables. Para el cálculo de los índices de precios al consumidor se utilizó el método de Laspayres. Para la comparación de éstos se utilizaron coeficientes de correlación de Pearson y de Sperman. Resultados. No se encontraron diferencias en los IPC por municipio ( $p>0.05)$. Los IPC acumulados año son de un dígito. Los porcentajes de inseguridad alimentaria (IA) estuvieron por encima de 50\%, encontrándose diferencias por municipio, en $1999(p=0.04)$, en $2000(p=0.88)$. La IA aumentó en promedio cinco puntos para el periodo 19992000. Se necesita en promedio 1.24 SMLV por mes para acceder a una CBA familiar. Conclusiones. La capacidad de compra del SMLV no satisface los requerimientos familiares de energía y nutrientes. La SA local seguirá deteriorándose, debido al comportamiento de sus determinantes. El texto completo en inglés de este artículo está disponible en: http://www.insp.mx/salud/index.html

Palabras clave: Sisvan; vigilancia nutricional; salario y beneficios; canasta alimentaría; C olombia

\author{
Herrán-Falla OF, Prada-Gómez GE, Patiño-Benavides GA. \\ Alimentary basic basket and index of prices \\ in Santander, Colombia, 1999-2000. \\ Salud Publica Mex 2003;45:35-42. \\ The English version of this paper \\ is available at: http://www.insp.mx/salud/index.html
}

\begin{abstract}
A bstract
Objective.To establish indicators for food security (FS) in two Colombian municipalities. Material and Methods. In 1999-2000, a descriptive study was carried out in two municipalities of the department of Santander, Colombia, that determined the cost of basic food baskets (BFB), to estimate price indices (PI), their variation, and indicators of FS related to the legally set minimum wage (MW ). Results. $\mathrm{N}$ o differences were found in the PI by municipality $(p>0.05)$. The annual cumulative price indices were a single digit.The percentages of food insecurity (FI) were upwards of $50 \%$, differing by municipality, in $1999(p=0,04)$, and $2000(p=0,88)$. The Fl increased five points on average for the period 1999 2000. An average minimum wage of 1.24 per month is needed for a family to have access to a BFB. Conclusions. The purchasing power of the current MW doesn't satisfy the caloric nor nutritional requirements of a family.The local FS will continue deteriorating, given the behavior of its determinants. The English version of this paper is available at: http://www.insp.mx/salud/index.html
\end{abstract}

Key words: Sisvan; nutritional surveillance; salaries and fringe; basket would feed; Colombia

(1) Escuela de Nutrición y Dietética. Universidad Industrial de Santander. Bucaramanga, Colombia.

(2) Escuela de Economía y A dministración. Universidad Industrial de Santander. Bucaramanga, Colombia.

Fecha de recibido: 11 de febrero de 2002 - Fecha de aprobado: 14 de septiembre de 2002

Solicitud de sobretiros: D r. 0 scar Fernando Herrán-Falla. C alle 33 N 0.29-14 A partamento 501. Bucaramanga, Santander, Colombia. Correo electrónico: herran28@epm.net.co 
E 1 desarrollo conceptual y metodológico de los sistemas de vigilancia alimentaria y nutricional (Sisvan), parte fundamental de la planificación y evaluación de políticas y estrategias nutricionales, ha sido apoyado por la Organización Panamericana de la Salud (OPS). En 1974, el Congreso Mundial de Alimentación recomendó el establecimiento de un sistema global para vigilar las condiciones de alimentación y nutrición de los grupos desfavorecidos. ${ }^{1}$ En América Latina empezaron a desarrollarse desde 1977.

En Colombia el Sisvan se estableció en abril de 1986; fue definido como un proceso permanente y oportuno de análisis e interpretación de la información generada en diferentes sectores, que permite la identificación de factores biológicos, ambientales, culturales y económicos que influyen en la malnutrición. ${ }^{2}$ El modelo adoptado está compuesto por tres componentes: estado de nutrición, estado de salud y seguridad alimentaria (SA). ${ }^{3}$

La SA es entendida como el acceso de todas las personas en todo momento a una alimentación que les permita una vida sana y activa; ${ }^{4}$ su objetivo, según el Comité de Seguridad Alimentaria Mundial de la Organización de las Naciones Unidas para la Agricultura y la Alimentación (FAO), es que todas las personas tengan en todo momento acceso físico y económico a los alimentos básicos que necesitan. 5,6

La SA familiar está condicionada principalmente por la disponibilidad y la demanda efectiva de los alimentos; ésta depende de los precios, el salario, las tasas de interés, el mercado de crédito, el tipo de cambio, el ingreso familiar, los activos del hogar, el ahorro y el autoconsumo (productores que consumen sus propios productos); otras variables determinantes en el país son el conflicto armado y la tasa de empleo. En Colombia y en el resto de América Latina, el deficiente acceso a los alimentos, debido a los bajos ingresos familiares, es el problema más importante que enfrenta la SA.,8

Mientras que los ingresos condicionan la cantidad de dinero destinado a la compra de alimentos, los precios determinan la cantidad de alimento que se puede comprar; por lo tanto, se debe relacionar el ingreso nominal, tomando como referencia el salario mínimo legal vigente (SMLV), con los precios de los alimentos, para conocer el acceso a ellos. Por esto se han utilizado como indicadores de la vigilancia en SA, el costo de la canasta básica de alimentos (CBA) y la capacidad de compra del SMLV (CC). ${ }^{9}$

La CBA es definida como el conjunto de alimentos suficientes para cubrir la necesidad energética, de proteína, hierro, calcio y vitamina A de una familia promedio ${ }^{10}$ lo que permite tomar decisiones sobre disponibilidad de alimentos básicos, promover la vigilancia de los precios, identificar la población en extrema pobreza, facilitar el diseño de políticas de bienestar nutricio y determinar la SA familiar. ${ }^{11-13}$

La CBA de este estudio fue diseñada en investigaciones previas de acuerdo con la metodología Sisvan, tomando en cuenta los hábitos alimentarios, las necesidades de energía y nutrientes de acuerdo con la edad, el sexo y la actividad física de los miembros de una familia tipo, y para satisfacer nutrientes considerados como problemas de salud pública en Colombia. La CBA se realizó a escala familiar, puesto que la familia es la unidad económica que determina el consumo individual. ${ }^{10,14-16}$

La información sobre los precios de la CBA y los ingresos familiares (teniendo como referencia el SMLV), permite calcular la CC, para conocer indirectamente el consumo de alimentos, la estabilidad de la oferta, el acceso que cada hogar tiene a la cantidad de alimentos que necesita y el nivel de vida de la población. . $^{16,17}$

En Colombia, para las ciudades capitales de departamento, se calcula el precio de la canasta básica familiar (CBF) que, además de los alimentos, incluye otros bienes y servicios y con él se construye el índice de precios al consumidor (IPC). Nuestros datos corresponden específicamente a los precios de los alimentos que hacen parte de la CBA en dos municipios en transición urbano rural (Piedecuesta y Girón), localizados cerca de Bucaramanga, la capital del departamento de Santander.

En América Latina el componente de SA no se ha logrado implantar en los Sisvan, debido a dificultades en el diseño y cuantificación de los indicadores respectivos. En la conferencia internacional de nutrición de 1992, se recomendó iniciar investigaciones y sistemas de vigilancia en alimentación y nutrición. ${ }^{11}$

El objetivo del estudio fue consolidar un componente del Sisvan, ${ }^{17-21}$-la vigilancia alimentaria local (VA)-, cuantificando el costo de las CBA en dos municipios, para establecer la CC del SMLV, determinar el IPC de la CBA y relacionarlo con el IPC DANE $_{\text {nacional y }}$ de Bucaramanga. Además, calcular los indicadores internacionales aceptados en materia de SA y enmarcarlos dentro de los contextos social, estadístico y alimentario nutricio.

\section{Material y métodos}

Con los datos que recolectó el sistema de VA, sobre el precio nominal de las CBA en los municipios de Piedecuesta y Girón, ${ }^{22}$ durante los años de 1999 y 2000 se realizó un estudio descriptivo prospectivo.

En cuatro diferentes sitios representativos de la oferta de alimentos, elegidos al azar para cada muni- 
cipio, se recolectaron semanalmente los precios de los alimentos que conforman las CBA. Cuatro de los ocho sitios de muestreo eran puntos de compra (micromercados), localizados en barrios clasificados por las oficinas de planeación municipal en los estratos uno a tres; los cuatro restantes fueron sitios de abastecimiento local, ampliamente conocidos y reconocidos como plazas de mercado. Todos los precios fueron recolectados los días de mercado por estudiantes del último año de la carrera de nutrición y dietética que se encontraban desarrollando proyectos de extensión en la comunidad, en Piedecuesta el día lunes, y en Girón el martes. Los datos fueron criticados en el campo y por los investigadores semanalmente.

Los registros fueron digitados por duplicado en bases relacionales diseñadas para la VA, en EpiInfo versión 6.04c y cruzados hasta descartar errores en el programa VALIDATE.

A partir de los precios de los alimentos se estimó en unidades de SMLV el costo semanal de las respectivas CBA. El valor del SMLV fue el reportado por el Departamento Administrativo Nacional de Estadísticas $(\mathrm{DANE})^{23}$ y es constante a lo largo del año. El costo mensual -en unidades de SMLV- $\left(\mathrm{UCBA}_{\text {mes }}\right)$ fue estimado como el promedio de las mediciones respectivas del mes.

Siguiendo la metodología del DANE, se calculó un índice $\left(\mathrm{IPC}_{\mathrm{PG}}\right)$ mediante el método de Laspayres. ${ }^{24}$ El valor obtenido con el cálculo del $\mathrm{IPC}_{\mathrm{PG}}$ en un periodo mide la relación, en términos porcentuales, entre el valor de la CBA a precios de dicho periodo y el valor de la misma a precios del periodo de base (mes inmediatamente anterior). Para esto se utilizó la siguiente ecuación;

$$
\mathrm{IPC}_{\mathrm{PG}}=\left(\left(\mathrm{UCBA}_{\mathrm{mes}}\left[\_\mathrm{n}\right] / \mathrm{UCBA}_{\mathrm{mes}}\left[\_\mathrm{n}-1\right]\right)-1\right) * 100
$$

Puesto que la base de comparación es 100, un valor de $\mathrm{IPC}_{\mathrm{PG}}$, superior a 100 reflejaría un aumento en el costo de la CBA. De la misma forma, un valor inferior reflejaría un descenso en dicho costo.

Para estimar la variación porcentual de los precios de las CBA en los meses del año y permitir su comparación con los índices de precios al consumidor oficiales $\left(\mathrm{IPC}_{\mathrm{DANE}}\right)$ para el país y Bucaramanga, ${ }^{25}$ fue calculado un $\mathrm{IPC}_{\mathrm{PG}}$ acumulado, con la fórmula:

$$
\sum \mathrm{IPC}_{\mathrm{PG}}
$$

Para estimar el indicador de inseguridad alimentaria teórica (IIAT), acogimos lo recomendado por el Sisvan, ${ }^{26,27}$ cálculo derivado del costo en pesos de la CBA (\$CBA) y la capacidad de compra de $80 \%$ del SMLV, con la fórmula:

$$
\mathrm{IIAT}=[(\$ C B A * 100) /(\mathrm{SMLV} * 0.80)]-100 .
$$

De esta forma, la inseguridad alimentaria es definida como la adecuación calórica que se podría comprar con $80 \%$ del SMLV. Un IIAT de 49.75 (cuadro I, mes de febrero, Piedecuesta), representaría un déficit teórico de $49.75 \%$ en las calorías necesarias para cubrir la adecuación en la familia tipo, dado el SMLV. Un valor de IIAT negativo o cercano a cero, sería ideal.

El análisis de los datos consistió en obtener líneas de tendencia durante el periodo estudiado para los valores del $\mathrm{IPC}_{\mathrm{PG}}$ y los del IPC $\mathrm{DANE}_{\mathrm{DAN}^{\prime}}$ mes a mes. Además, se compararon los $\mathrm{IPC}_{\mathrm{PG}}$ a través de pruebas $t$ de student ${ }^{28}(\alpha 0.05)$ y se obtuvieron coeficientes de correlación de Spearman ${ }^{28,29}$ entre los IPC $_{\mathrm{PG}}$ y los $\mathrm{IPC}_{\mathrm{DANE}}$, con el fin de establecer si existe independencia entre las mediciones realizadas en los municipios de Piedecuesta y Girón (VA) y las realizadas por el DANE para Bucaramanga y el país. El procesamiento estadístico de los datos fue realizado con el programa Stata.*

Este estudio no presentó ningún riesgo real o potencial para las poblaciones participantes. No tomó ni manipuló información de sujetos. El comité de ética de la Facultad de Salud de la Universidad Industrial de Santander aprobó la realización del estudio.

\section{Resultados}

Para el municipio de Piedecuesta fueron vigiladas las CBA de 22 meses, y para el de Girón de 23, calculándose n-1 IPC $\mathrm{IPG}_{\mathrm{PG}} \mathrm{Al}$ explorar la forma en la cual se distribuyeron los IPC $\mathrm{PG}_{\mathrm{PG}}$ y los $\mathrm{IPC}_{\mathrm{DANE}^{\prime}}$ se encontró que sólo los $\mathrm{IPC}_{\mathrm{PG}}$ adoptan una función de probabilidad normal ${ }^{\ddagger}$ ( $p>0.05$;test de Shapiro-Wilk).

No se encontraron diferencias estadísticamente significativas entre los $\mathrm{IPC}_{\mathrm{PG}}$ calculados para los municipios por año; 1999 ( $p=0.8300), 2000(p=0.6960)$, ni en su conjunto $(p=0.6649)$. A diferencia de lo anterior, al comparar el IIAT para el año 1999, se encontró diferencia estadísticamente significativa, con desventaja para el municipio de Girón ( $p=0.0385)$, pero en el año 2000 no $(p=0.8816)$. El cuadro I muestra la evolución del costo en pesos (\$)/día, de la CBA y del IIAT por año, mes y municipio. Esta, además, muestra una inte-

\footnotetext{
* StatCorp. 2001. Stata Statistical Software: Release 7.0. College Station, TX: Stata Corporation.

¥ Shapiro-Wilk and Shapiro-Francia test. Stata Technical Bulletin sg3.2, 1991; 3:19. Reprinted in Stata Technical Bulletin Reprints, 1991c;vol.1:105.
} 
Costo/día* de la Canasta Básica de Alimentos y PORCENTAJE TEÓRICO DE INSEGURIDAD ALIMENTARIA, SEGÚN SISTEMAS DE VIGILANCIA ALIMENTARIA Y nutricional. Piedecuesta y Girón, Santander, Colombia, 1999-2000

\begin{tabular}{|c|c|c|c|}
\hline & Piede & desta & Girón \\
\hline Mes & $\begin{array}{c}\text { Costo CBA } A^{\ddagger} \\
(\$) / \text { día }\end{array}$ & $\begin{array}{l}\text { \% teórico de } \\
\text { inseguridad } \\
\text { alimentaria }\end{array}$ & $\begin{array}{c}\text { Costo CBA } \\
(\$) / d i ́ a\end{array}$ \\
\hline
\end{tabular}

\begin{tabular}{lcccc}
$\begin{array}{l}\text { Año } 1999 \S \\
\text { Febrero }\end{array}$ & 9442.63 & 49.75 & 9861.21 & 56.39 \\
\hline Marzo & 9541.78 & 51.32 & 9585.92 & 52.02 \\
\hline Abril & 9317.80 & 47.77 & 9526.50 & 51.08 \\
\hline Mayo & 9426.03 & 49.49 & 9628.73 & 52.70 \\
\hline Junio & 9282.94 & 47.22 & 9627.70 & 52.68 \\
\hline Julio & 9386.94 & 48.87 & 9757.00 & 54.73 \\
\hline Agosto & 9149.25 & 45.10 & 9660.75 & 53.21 \\
\hline Septiembre & 9072.81 & 43.88 & 9171.87 & 45.46 \\
\hline O ctubre & 9157.87 & 45.23 & 9390.47 & 48.92 \\
\hline Noviembre & 9702.95 & 53.88 & 9733.47 & 54.36 \\
\hline Diciembre & 9958.50 & 57.93 & 10124.18 & 60.56 \\
& & & & \\
Promedio & 9403.51 & 49.13 & 9642.53 & 52.92 \\
\hline (DS) & $(259.87)$ & $(4.12)$ & $(245.69)$ & $(3.90)$
\end{tabular}

\begin{tabular}{lcccc}
$\begin{array}{l}\text { Año } 2000^{\S} \\
\text { Enero }\end{array}$ & 10475.78 & 51.03 & 10540.92 & 51.97 \\
\hline Febrero & 10831.05 & 56.16 & 10818.77 & 55.98 \\
\hline Marzo & 10914.31 & 57.36 & 10954.24 & 57.93 \\
\hline Abril & 11307.13 & 63.03 & 11168.59 & 61.02 \\
\hline Mayo & 10942.85 & 57.77 & 11057.47 & 59.42 \\
\hline Junio & 10755.63 & 55.07 & 10839.13 & 56.27 \\
\hline Julio & 10720.50 & 54.56 & 10916.00 & 57.38 \\
\hline Agosto & 10718.88 & 54.54 & 10790.48 & 55.57 \\
\hline Septiembre & 10704.67 & 54.33 & 10701.10 & 54.28 \\
\hline O ctubre & 10680.50 & 53.99 & 10578.60 & 52.52 \\
\hline N oviembre & 10597.84 & 52.79 & 10508.68 & 51.51 \\
\hline Diciembre & 10475.78 & 51.03 & 10389.72 & 49.79 \\
& & & & \\
Promedio & 10786.29 & 55.51 & 10771.98 & 55.30 \\
\hline (DS) & $(216.17)$ & $(3.14)$ & $(235.69)$ & $(3.40)$
\end{tabular}

* En mayo 22 de 2001, por \$2 327 se compraba un dólar americano.

₹ CBA:C anasta básica de alimentos

§ En 1999 el valor del salario mínimo legal vigente/día: \$7 882. En 2000 el valor del SMLV/día: \$8 670

resante relación entre la tendencia de los precios de la CBA y el IIAT; mientras que en Piedecuesta la tendencia creciente de los precios se corresponde con una tendencia creciente en el IIAT, en Girón una relativa estabilidad de los precios se corresponde con una tendencia decreciente en el IIAT.
El cuadro II muestra los IPC $\mathrm{PG}_{\mathrm{PG}}$ IPC $\mathrm{DANE}_{\text {, }}$ acumulados para los años 1999 y 2000 y su variación en el periodo, para la CBA y la CBF, en diferentes estratos socioeconómicos; éstos son equivalentes a la inflación cursada durante el año. Al comparar los IPC acumulados del año 2000 versus 1999 en la CBA, se observó una variación negativa -disminuyeron los precios de alimentos-, para los dos municipios estudiados, el estrato alto nacional y el país. Lo mismo sucedió en la CBF del país y de Bucaramanga.

En el cuadro III, se observa que los IPC $\mathrm{PG}_{\mathrm{PG}}$ IPC $\mathrm{DA}_{\mathrm{DA}}$ ${ }_{\mathrm{NE}}$ mensuales, no son diferentes, debido a los coeficientes de correlación calculados, excepto para el estrato alto del nivel nacional en la CBA y la CBF.

La figura 1 muestra las tendencias del SMLV/día y su CC de la CBA [1/SMLV] para los dos años y municipios vigilados; la relación está invertida contra una teórica deseada. ${ }^{27} \mathrm{La}$ distancia entre las dos líneas calculadas es la brecha a corregir para acercarse a un IIAT de cero o negativo.

La figura 2 muestra las líneas de tendencia de los

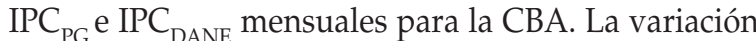

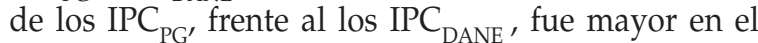
primer año vigilado que en el segundo.

Cuadro II

INDICE DE PRECIOS AL CONSUMIDOR ACUMULADO AÑO, DE LA VIGILANCIA ALIMENTARIA LOCAL (Piedecuesta y Girón), y nacional. Santander, Colombia, 1999-2000

Año

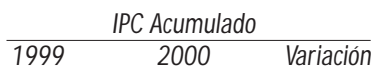

Canasta básica de alimentos

\begin{tabular}{llrr}
$I P C_{P G}$, Piedecuesta & $6.74 *$ & 6.55 & -0.19 \\
\hline $\mathrm{IPC}_{\mathrm{PG}}$, Girón & $3.59^{*}$ & 2.78 & -0.81 \\
\hline Bucaramanga & 6.14 & 10.28 & +4.14 \\
\hline Estrato bajo & 6.12 & 10.12 & +4.00 \\
\hline Estrato medio & 6.22 & 10.20 & +3.98 \\
\hline Estrato alto & 5.74 & 11.45 & +5.75 \\
\hline N acional & 7.49 & 7.41 & -0.08 \\
\hline Estrato bajo & 7.33 & 7.43 & +0.10 \\
\hline Estrato medio & 7.35 & 7.50 & +0.15 \\
\hline Estrato alto & 8.67 & 6.93 & -1.74
\end{tabular}

Canasta básica familiar

$\begin{array}{lrrr}\text { Bucaramanga } & 10.11 & 9.59 & -0.52 \\ \text { N acional } & 9.23 & 8.74 & -0.49\end{array}$

* Enero y febrero fueron estimados como el promedio de los 10 meses restantes del año en curso

IPC: Indice de precios al consumidor

salud pública de méxico / vol.45, no.1, enero-febrero de 2003 


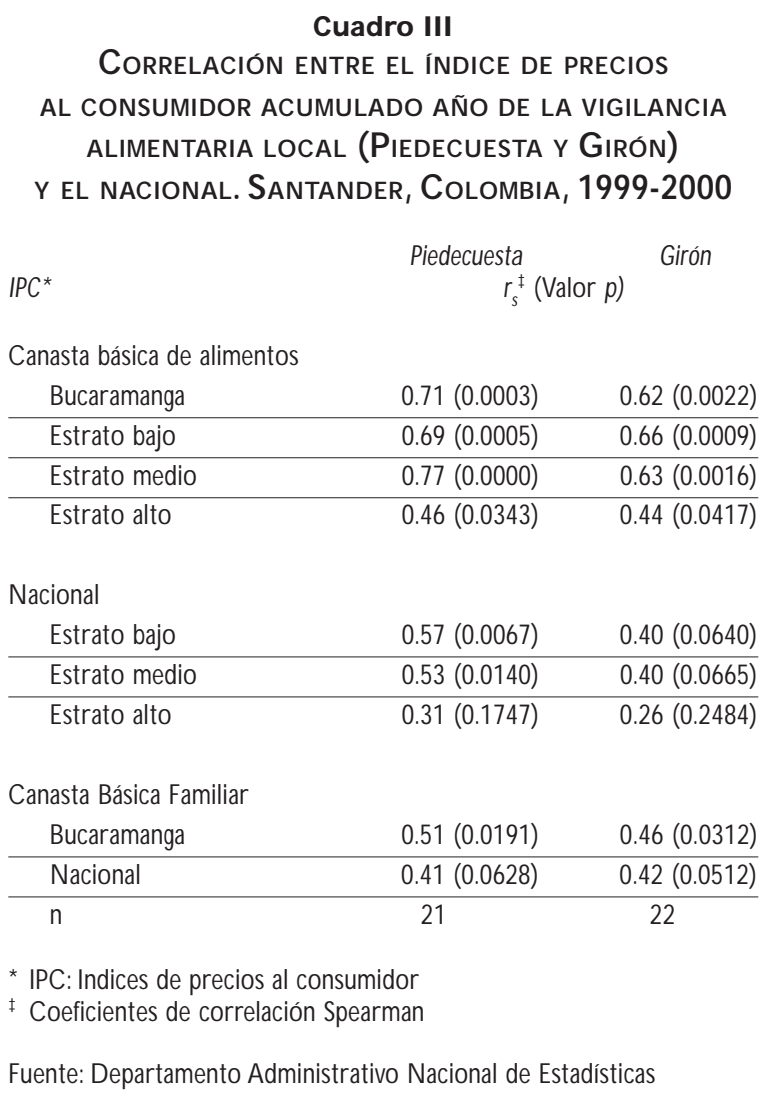

\section{Discusión}

Aspectos sociales. En la última década la SA, como otros indicadores de tipo social, se ha deteriorado tan rápidamente que el programa de las Naciones Unidas para el Desarrollo relegó a Colombia en su informe de $1999^{30}$ del lugar 54 al 57 en la escala de Desarrollo Humano.

A pesar de que la variación acumulada año en el IPC (cuadro II), ha logrado situarse en un solo dígito, la SA no mejorará en el corto y mediano plazo, ya que el ajuste del SMLV en la última década se ha hecho por debajo del IPC acumulado año, y que otras variables determinantes de la SA, como el empleo y el ingreso en los sectores más pobres de la población, siguen en deterioro. Según el Banco Mundial, Colombia es el segundo país de mayor concentración de la riqueza en el mundo y presenta la mayor tasa de desempleo del continente americano $(22 \%){ }^{31}$ Según las encuestas de hogares del DANE, el índice Gini de concentración del ingreso por persona pasó de 0.55 en 1991 a 0.56 en 1995. Para 1997, se estimó que este indicador llegó al $0.57^{32}$ (el índice Gini toma valores entre 0 y 1 , aumenta en la medida que haya concentración del ingreso). En el cua- dro II, el fenómeno de concentración se ilustra en la variación de los IPC acumulados año de la CBA; mientras que para el estrato alto nacional la variación es negativa, para los estratos bajo y medio nacional es positiva (los aumentos en el IPC perjudican a las familias pobres, quienes destinan un mayor porcentaje de sus ingresos para adquirir alimentos). Complementariamente, desde el año 1995, en el que se celebró la Cumbre Social, la pobreza ha aumentado persistentemente: de $55.1 \%$ en 1995 pasó a 57.0 en $1997^{32-36}$ y a 60 en 1999.30

Hay otro elemento que influye directamente en detrimento de la SA: la guerra y sus consecuencias. Según la Defensoría del Pueblo, desde 1985 hasta el primer semestre de 1998, han sido desplazados más de 1300000 colombianos, con un promedio de 100000 personas por año; Santander y estos dos municipios presentan altas tasas de recepción. ${ }^{37}$ Este tipo de perturbaciones internas alteran el crecimiento económico, el poder adquisitivo de la población, los patrones de consumo de alimentos, y el funcionamiento del mercado, con las repercusiones propias en la producción, comercialización y precios de los alimentos.

Aspectos estadísticos. En este estudio para medir el grado de asociación entre los IPC $\mathrm{PG}_{\mathrm{PG}} \mathrm{y}$ los IPC $\mathrm{DANE}_{\mathrm{DAN}}$ mensuales se utilizaron, por dos razones, coeficientes de correlación de Spearman: por la anormalidad de la distribución de los $\mathrm{IPC}_{\text {DANE }} \mathrm{y}$ por el tamaño muestral $(n=22)$. Estas dos condiciones limitaron el uso del coeficiente de Pearson y obligaron al uso del de Spearman, permitiéndose examinar asociaciones de una forma más general ${ }^{29}$-no lineales-. Los valores de $p<0.05$ (cuadro III) sugieren que para realizar la VA podrían ser utilizados casi todos los $\mathrm{IPC}_{\mathrm{DANE}^{\prime}}$ en reemplazo de los $\mathrm{IPC}_{\mathrm{PG}}$ (figura 2) Lo anterior permitiría, considerada la dificultad de cuantificar este indicador en la VA, utilizar los $\mathrm{IPC}_{\mathrm{DANE}}$ en el Sisvan y como herramienta de planificación municipal. ${ }^{20-22,38}$

Aspectos nutricionales. La visión de la seguridad alimentaria ha evolucionado de una mirada nacional centrada en el suministro de alimentos, a una localizada en el ámbito familiar y su acceso alimentario. Este enfoque es una herramienta eficaz para diseñar las políticas alimentarias. ${ }^{39}$

Los principales factores del acceso a los alimentos son los precios de los alimentos y los ingresos; éstos complementariamente aumentan o limitan las posibilidades de acceso a una seguridad alimentaria y nutricional, y condicionan la cantidad y calidad de alimento consumido. ${ }^{17,40}$ En Colombia para 1990, 67\% de los habitantes del área rural y $42 \%$ de los de la urbana, no disponían de ingresos suficientes para 

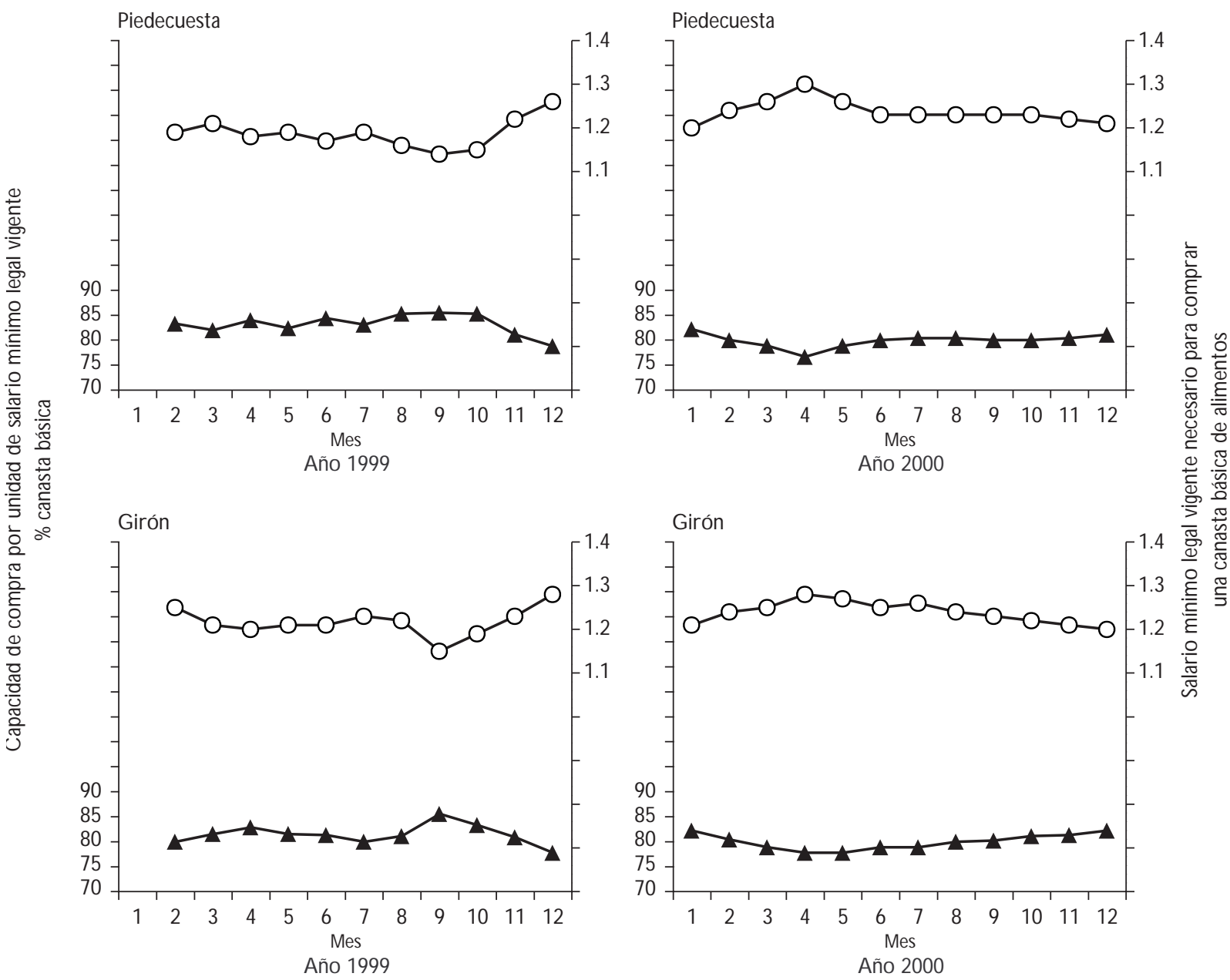

Figura 1. Salario y capacidad de compra. Santander, Colombia, 1999-2000

adquirir una canasta de básicos, incluidos los alimentos. ${ }^{41}$

Además, los ingresos determinan el gasto en alimentos, existiendo una relación inversa entre la proporción del ingreso que se destina al gasto en alimentos y el aumento de éste, ${ }^{42}$ de esta manera se podría concluir que el gasto en alimentos es regresivo. Dicho gasto en Colombia para 1994 fue de $41.44 \%$ en el estrato socioeconómico más bajo, y de $14.69 \%$ en el más alto; ${ }^{43}$ en Bucaramanga, $38.89 \%$ y $16.32 \%$ respectivamente. Llama la atención que durante el periodo de 1953 a 1994 el gasto en alimentos se redujo en ocho puntos, tanto para el estrato bajo como para el medio. ${ }^{43,44}$

Utilizando la información sobre precios e ingresos se construye el indicador de CC, para medir indirectamente el consumo de alimentos en la población y analizar el nivel de vida de la misma. ${ }^{45} \mathrm{El}$ compor- tamiento de la CC en nuestra región no permite satisfacer las necesidades alimentarias ${ }^{46}$ (figura 1).

La principal causa de inseguridad alimentaria para América Latina es la dificultad para acceder económicamente a los alimentos. ${ }^{7,11,47}$ En Colombia, para el periodo 1961-1971, 38\% de la población no tenía acceso suficiente a los alimentos, situación que mejoró para el periodo 1990-1992, situándose en $19 \% .{ }^{48}$ En este estudio el IIAT mostró un deterioro en promedio de cinco puntos de un año a otro (cuadro I); este indicador en 1993 para la región fue de 50.32. ${ }^{49}$

El valor de la CBA en Centroamérica se utiliza para determinar el indicador de CC de la población; en 1994, en Belice se necesitaban dos SMLV para adquirir una CBA, en Costa Rica 1.19, en Nicaragua 0.50, en Honduras 0.34 y en Guatemala 0.22. ${ }^{50}$ En 1995, en Bucaramanga se necesitaban $1.15 \mathrm{SMLV}$ para acceder a la CBA, 


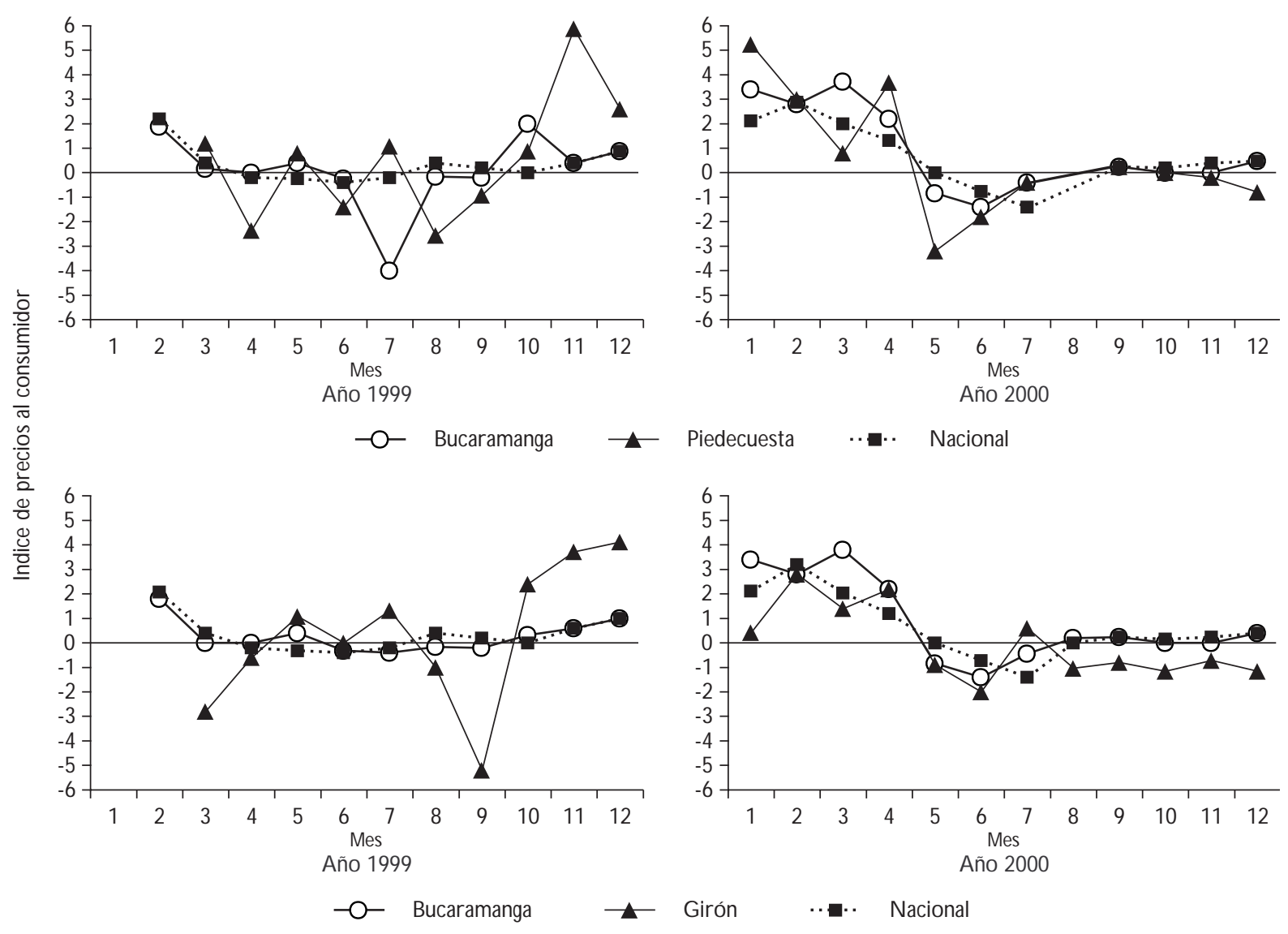

Figura 2.Variación en el Indice de precios al consumidor de los alimentos. Colombia, 1999-2000

mientras que en 1997, 1.07. ${ }^{44}$ En Piedecuesta y Girón para 1999 se compraba una CBA con 1.19 y $1.24 \mathrm{SMLV}$, respectivamente; para el año 2000 este indicador alcanzó 1.24 para los dos municipios (figura 1). Si aceptamos que una unidad familiar colombiana (cinco miembros), para satisfacer sus requerimientos nutricionales gasta alrededor de $40 \%$ de su ingreso, ${ }^{43,44}$ nuestra unidad familiar requeriría de por lo menos tres SMLV, para satisfacer sus necesidades más básicas (vivienda, ropa, etc.). Considerados la creciente concentración de la riqueza, el aumento de los indicadores de pobreza, desempleo y conflicto armado, ${ }^{30-37}$ esto cada vez es menos probable, y nuestros hallazgos son una demostración explícita de inequidad.

Todo lo hasta aquí discutido refuerza la necesidad de una política pública, desde las regiones, que apunte al bienestar de la población, con indicadores como los derivados por el SISVAN, que incorporan no sólo la racionalidad que opera tras nuestra visión instrumental de los procesos, sino la historia y la cultura -alimentaria en este caso- de nuestras clases subalternas, máxime, si como fue demostrado, un IPC acumulado año de un dígito no necesariamente garantiza una mejoría en el IIAT.

\section{Referencias}

1. O rganización Panamericana de la Salud/O rganización Mundial de la Salud. Vigilancia alimentaria y nutricional en las Américas. Una Conferencia Internacional. México, 5-9 de septiembre de 1988.W ashington, D.C .: O PS, 1989; Publicación científica N 0. 516:216.

2. Montoya CH, G acharná MG,Vargas E,Villate M. El sistema de vigilancia epidemiológica alimentaria y nutricional en Colombia. En: O rganización Panamericana de la Salud/O rganización Mundial de la Salud.Vigilancia alimentaria y nutricional en las Américas. Una Conferencia Internacional. México, 5-9 de septiembre de 1988.W ashington, D.C.:O PS, 1989; Publicación científica N 0. 516:125-132.

3. Departamento $\mathrm{N}$ acional de Planeación. Sistema de vigilancia epidemiológica alimentaria y nutricional. Manual de capacitación. SISVAN . Bogotá: Editorial Gente N ueva, 1987;fascículo 5.

4. 0 rganización de las $\mathrm{N}$ aciones Unidas para la A gricultura /O rganización Mundial de la Salud. Elementos principales de estrategias nutricionales. 
Mejora de la seguridad alimentaria en los hogares. Roma: Conferencia internacional de nutrición, 1992:2-43.

5. $O$ rganización de las $\mathrm{N}$ aciones Unidas para laA gricultura. Cumbre mundial sobre la alimentación. 13-17 de noviembre de 1996. Roma: ONU, 1996:1-49.

6. G arcía C, C rovetto M. Situación de la seguridad alimentaria en América Latina. Rev Chil N utr 1991;19:17-24.

7. Declaración en defensa de la seguridad alimentaria por parte de las organizaciones de la sociedad civil latinoamericanas y del Caribe. A sunción, Paraguay, 1996.

8. Sharma RP. Monitoring access to food and household food security. Food N utr Bull 1992; 2:2-9.

9. Durán JR. Incremento inmoderado de canasta básica. Tegucigalpa, Honduras: Secretaría de Asistencia técnica y C ooperación Internacional, 1996. 10. Herrán O F, Prada GE. D eterminación de la canasta básica alimentaria de dos municipios colombianos. Salud UIS 1999:30:22-29.

11.0 rganización de las $\mathrm{N}$ aciones U nidas para laA gricultura. Estado actual y perspectivas de la vigilancia alimentaria y nutricional en A mérica Latina y el Caribe. Santiago de Chile: FAO, 1994:295.

12. Ministerio de Salud, Departamento de Nutrición. Plan Nacional de Seguridad Alimentaria N utricional de C osta Rica, 1997-2001. San José de Costa Rica: Impresos Litográficos, 1997. Disponible en: http:// www.brssan.incap.org.gt/scripts/wxis.exe/iah/. C onsultado en octubre de 2002.

13. May E. La pobreza en Colombia. Un estudio del Banco Mundial. Bogotá: Editorial TM/ Banco Mundial, 1995:3-12.

14. Mora 0. Situación nutricional de la población colombiana en 1977-80. Bogotá: Instituto Nacional de Salud, julio 1982; vol. 1.

15. Yepes FJ. La salud en Colombia. D ocumento General.Tomo II. Bogotá: Ministerio N acional de Salud/D epartamento N acional de Planeación, 1990. 16. Departamento $\mathrm{N}$ acional de Planeación. Plan nacional de alimentación y nutrición. 1996-2005. Bogotá: DN P, 1996.

17. Prada GE. Hábitos alimentarios de las madres residentes en la vereda Chocoita del municipio de Girón (tesis). Bucaramanga: Pontificia U niversidad Javeriana, U niversidad Industrial de Santander, 1996.

18. Herrán OF, Prada GE, Patiño GA. Condicionantes socioeconómicos de la ingesta de energía en mujeres adultas. Salud UIS 1999;30(1):3-9.

19. Herrán O F, Prada GE. Situación alimentaria en mujeres de 2 municipios Santandereanos. Salud UIS 2001;33(2):134-141.

20. Herrán O F,Torres 0 . Diagnóstico de la situación de salud, alimentaria y nutricional de un sector de la población del municipio de Piedecuesta. Bucaramanga: Universidad Industrial de Santander, 1998.

21. Prada GE. Diagnóstico de la situación alimentaria y nutricional del municipio de Girón. Bucaramanga: Universidad Industrial de Santander, 1998.

22. Sistema de recolección de precios de canasta básica alimentaria ajustada. Piedecuesta y G irón. Escuela de N utrición y D ietética. Bucaramanga: Universidad Industrial de Santander, 1997.

23. D epar tamento $\mathrm{N}$ acional de Planeación. División de indicadores y orientación del gasto social. República de Colombia: DN P, 2001.

24. Departamento Administrativo Nacional de Estadística. Metodología índice de precios al consumidor IPC -98. Bogotá: DAN E, 1998:35-43.

25. Departamento Administrativo $\mathrm{N}$ acional de Estadística. Indice de precios al consumidor. Bogotá: DAN E. D isponible en:http://www.dane.gov.co. 26. Departamento Administrativo $\mathrm{N}$ acional de Estadística. Indice de precios al consumidor-Información correspondiente a los años 1999 a 2000. Disponible en: http://www.danegov.co.

27. Universidad delValle. Módulo alimentos y dieta. En:Vigilancia nutricional y seguridad alimentaria. N utrición- salud-dieta. C ali:UV, 1994:iv2-iv15. 28. Fajardo P, Lareo L,A cciarri G, PradillaA, et al. Módulo alimentos y dieta. En:Vigilancia nutricional y seguridad alimentaria. N utrición-salud-dieta. Cali: Universidad del Valle, 1994;iv2-iv15.
29.Altman D G. Practical statistics for medical research. N ueva York (N Y): Editorial Chapman \& Hall/CRC, 1999:277-299.

30. Programa de las $\mathrm{N}$ aciones Unidas para el Desarrollo. Informe sobre desarrollo humano. Bogotá, Colombia: PN UD, 1999.

31. Sarmiento L. Exclusión, conflicto y desarrollo social. Bogotá: Editorial Desde A bajo, 1999:104.

32. Sarmiento L. El desarrollo social en la encrucijada. En: Síntesis 98. Anuario social, político y económico de Colombia. Bogotá: Editorial TM, 1998: 23-33.

33. El desempleo no da tregua. El tiempo 1998;octubre 20 sección B:1,7. 34. Consejería Presidencial para la Política Social - Plan N acional de Rehabilitación- Programa de las $\mathrm{N}$ aciones Unidas para el D esarrollo. Informe de Colombia a la C uarta Conferencia Internacional de la Mujer en Beijing. China, 1995. Bogotá: Presidencia de la República, 1995.

35. Cumbre social contra la pobreza, por la equidad y la paz. D ocumento de las comunidades negras. Bogotá: Editorial Sampher, 1998:l.

36. Banco Mundial. La hora de la reforma. Bogotá:Tercer Mundo Editores, 1998.

37. Comentarios y propuestas sobre la situación de los derechos económicos, sociales y culturales y el desplazamiento forzado en Colombia. Exodo 1998:9:3-21.

38. O rganización de las $\mathrm{N}$ aciones Unidas para la Agricultura. Manejo de Proyectos de Alimentación y N utrición en Comunidades. Guía didáctica. roma: O NU, 1995:205-229.

39. 0 rganización de las $\mathrm{N}$ aciones Unidas para la Agricultura. Programa Mundial de Alimentos.Tiempo de cambiar: La ayuda alimentaria y el desarrollo. Seguridad alimentaria, medios de subsistencia e intervenciones de ayuda alimentaria. Roma: 0 N U, 1988:23-24.

40. Sarmiento E. Funcionamiento y control de una economía en desequilibrio. Bogotá: Editorial CEREC, 1987:175-298.

41. Sarmiento L. La pobreza rural en Colombia. En: Pérez Edelmira, comp. El campesinado en Colombia hoy. Bogotá: Editorial Ecoe, 1991: 17-47.

42. Espinosa F,Valiente G,Valiente S. SISVAN de alimentos índides. Santiago de Chile: Universidad de Chile, 1996.

43. D epartamento Administrativo $\mathrm{N}$ acional de Estadística. Indice de Precios al Consumidor. Encuestas de ingresos y gastos. IPC 20, IPC 40, IPC 60. Encuesta 1994-1995. Colombia: DANE, 1996.

44. Corzo E, Maecha M, Poveda G, Prada GE, Rueda EO. Indicador de seguridad alimentaria en Bucaramanga. 1995-1996. SISVAN Santander. Bucaramanga: Secretaría de Salud de Santander, 1997.

45. Martorell R, Habicht JP. Indicadores nutricionales en los sistemas de vigilancia alimentaria y nutricional. En: organización Panamericana de la Salud/O rganización Mundial de la Salud.Vigilancia A limentaria y N utricional en las A méricas.W ashington, D C: O PS/O MS, 1989; Publicación Científica N 0. 516:55-70.

46. Laure J, Menchú MT, Batres R. Elementos para el cálculo del salario mínimo en Guatemala. Guatemala: IN CAP, 1994:24.

47. 0 rganización de las $\mathrm{N}$ aciones Unidas para la Agricultura. Sistema de información y cartografía, so bre inseguridad y vulnerabilidad alimentarias. SICIVA. Disponible en: http://www.fivims.net.2000. Consultado en abril de 2000.

48. Fajardo L. Perfil nutricional por país. Colombia: 0 rganización de las Naciones Unidas para la Agricultura 1998. Disponible en: http:// www.fao.org. Consultado en abril de 2000.

49. Unidad Regional de Planificación Agrícola. Análisis de seguridad alimentaria en Santander.Bucaramanga: Secretaría de Salud de Santander, SISVAN , 1994:6-17.

50. Laure J. Salario mínimo, seguridad alimentaria y pobreza. Guatemala: Instituto de Nutrición de Centroamérica y Panamá, 0 rganización Panamericana de la Salud, 1988;D ocumentos técnicos N 0. 27:67. 